\title{
Estudio de la composición química del aceite esencial de orégano (Origanum vulgare spp.) de Tacna
}

\author{
Lena A. Tellez Monzón* \\ Diana María Nolazco Cama* \\ Universidad Nacional Agraria La Molina. Lima, Perú \\ Recibido: 10 de enero del 2017 / Aprobado: 23 de agosto del 2017
}

REsumen: Se analizó químicamente el aceite esencial de orégano (Origanum vulgare spp.) cultivado en la ciudad de Tacna (Perú). El análisis fisicoquímico determinó las siguientes características: color ( $\left.L^{*} 60,51, a^{*}-2,83, b^{*} 14,31\right)$, índice de refracción de 1,475; densidad específica de 0,9132, y solubilidad en alcohol de $75 \mathrm{ml}$. Asimismo, la composición química se evaluó mediante cromatografía de gases, acoplada a espectrometría de masas. El cromatograma mostró los siguientes porcentajes: 1 - 4 terpineol, 26,56; timol, 18,80; $\gamma$-terpineno, 11,77; 2 -careno, 6,53; terpineol, 4,08; m-cimeno, 3,27; y carvacrol, 2,24, entre otros presentes en menor proporción.

Palabras clave: orégano / cromatografía / composición química / aceite esencial

\section{Study on chemical composition of the oregano essential oil (Origanum vulgare spp.) from Tacna}

AвstRACT: The oregano essential oil (Origanum vulgare spp.) cultivated in the city of Tacna, Peru, has been chemically analyzed. The physicochemical analysis determined the following characteristics: color (L * 60,51, $a *-2,83, b * 14,31)$, refractive index: 1,475 ; specific density: 0,9132 and solubility in alcohol: $75 \mathrm{ml}$. Also, the chemical composition was evaluated by gas chromatography coupled to mass spectrometry. The chromatogram showed the following percentages: 1-4 terpineol with 26,56; thymol with 18,80; $\gamma$-terpinene, 11,77; 2-carene, 6,53; Terpineol, 4,08; M-cymene, 3,27 and carvacrol 2,24, among others present in smaller proportion.

Keywords: oregano / chromatography / chemical composition / essential oil

* Correos electrónicos: ltellez@lamolina.edu.pe,dnolazco@lamolina.edu.pe 


\section{INTRODUCCIÓN}

Origanum vulgare pertenece a la familia de las Lamiaceae. Es originaria de Eurasia occidental y el suroeste y la región mediterránea, y se encuentra distribuida en los cinco continentes (Banchio et al., 2008). El orégano puede ser diez veces más rentable que otros productos tradicionales, como el maíz amiláceo, la chala y la alfalfa -que requieren abundante cantidad de agua-, por lo que es una alternativa para las zonas altoandinas más pobres, como Candarave, Jorge Basadre y Tarata en la región Tacna (Maximixe, 2007).

La característica más importante de un aceite esencial son los monoterpenos oxigenados, sobre todo porque este aceite se compone de diferentes grupos funcionales, como alcoholes, aldehídos, cetonas, éteres, etc. Químicamente, los aceites esenciales están formados por terpenos, monoterpenos y sesquiterpenos (hidrocarburos, alcoholes y cetonas, que pueden ser acíclicos, monocíclicos, bicíclicos a tricíclicos), y sustancias azufradas y nitrogenadas (Govindarajan, Rajeswarya, Hotib y Benellic, 2016). Se ha estudiado la composición de los aceites esenciales de muchas especias y hierbas, en particular las de la familia Lamiaceae -a la que pertenece el orégano-, siendo de interés el sabor o flavor; sin embargo, el aceite escencial puede aplicarse con éxito en otros ámbitos.

Entre los compuestos principales reportados en estudios previos (Govindarajan et al., 2016) se encuentran el carvacrol (38,30 \%), y el terpineno-4-ol (28,70 \%). Sus estructuras químicas se muestran en la figura 1.

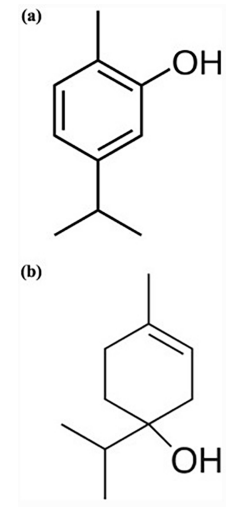

Figura 1. Estructura química de dos componentes principales de aceite esencial de Origanum vulgare spp.: carvacrol (a), terpineno-4-ol (b) Fuente: Govindarajan (2016) 
Ha crecido de manera importante la producción de orégano en el sur del Perú, en los departamentos de Tacna, Moquegua, y Arequipa. Específicamente, Candarave (distrito de Tacna) es el de mayor producción y exportación en los últimos años. En vista de ello, en esta investigación se ha produrado caracterizar los perfiles químicos y fisicoquímicos del aceite esencial de Origanum vulgare spp. procedente de esta región.

\section{PARTE EXPERIMENTAL}

La investigación fue realizada en la Universidad Nacional Agraria La Molina (UNALM), en los laboratorios del Departamento Académico de Química de la Facultad de Ciencias y en los laboratorios de Fisicoquímica e Investigación de la Facultad de Industrias Alimentarias.

\subsection{Metodología}

Para el presente estudio se elaboró el diagrama de bloques, el cual se describe en la figura 2.

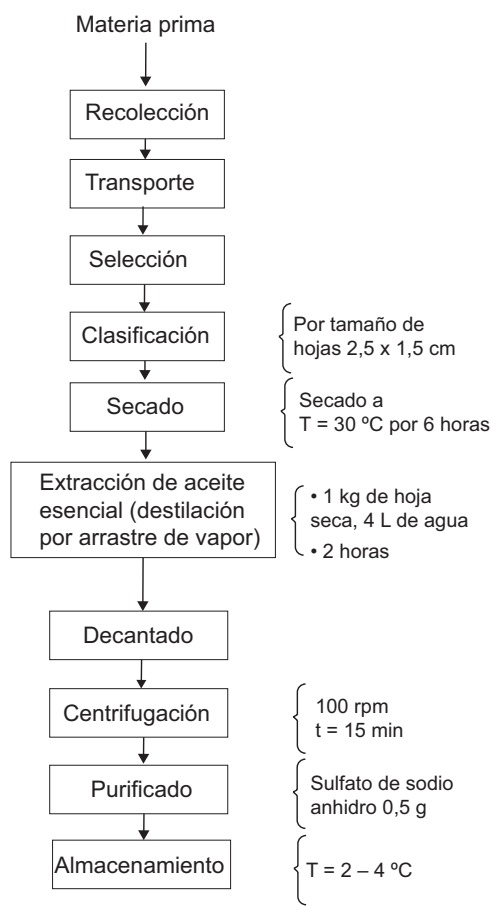

Figura 2. Diagrama de bloques de la extracción de aceite esencial de orégano Elaboración propia 


\subsubsection{Toma de muestra}

Se obtuvieron 20 muestras de hojas de orégano (Origanum vulgare spp.) seleccionadas por un muestreo aleatorio simple, del mercado de la ciudad de Tacna.

\subsubsection{Obtención de aceite esencial de orégano}

Las muestras de hojas orégano se clasificaron según su tamaño $(2,5 \mathrm{~cm}$ x $1,5 \mathrm{~cm}$ ), luego se desecaron en un secador a $30{ }^{\circ} \mathrm{C}$ por seis horas y se molieron para la destilación por arrastre de vapor de agua. El aceite se separó de la capa acuosa con pera de decantación, se secó con sulfato de sodio y se filtró. Con el producto se precisaron el color, índice de refracción, índice de acidez, densidad específica, solubilidad en etanol y rendimiento. La densidad o gravedad específica se determinó con un picnómetro; el índice de refracción se estableció utilizando un refractómetro Abbé Bausch \& Lomb modelo 31; el color del aceite, con un colorímetro Chromameter CR200® Minolta; el rendimiento se calculó al dividir la cantidad de aceite que se obtuvo de la muestra vegetal entre el peso de masa seca de la misma muestra. Todas las determinaciones se realizaron por triplicado. Una alícuota del aceite esencial de orégano (AEO) de la muestra que presentó el mayor rendimiento se envió al cromatógrafo de gases para determinar su composición química por medio de una técnica que combinó la capacidad de separación de la cromatografía de gases con la sensibilidad y capacidad selectiva del detector de masas (GC-MS).

\subsubsection{Análisis cromatográfico del aceite esencial de orégano}

Se necesitaron $20 \mu \mathrm{l}$ de aceite esencial para el análisis cromatográfico y se aforó a $450 \mu \mathrm{l}$ con diclorometano (grado qp). La mezcla se realizó en una fiola de $2 \mathrm{ml}$, después se transfirió a un vial para cromatografía de gases de $2 \mathrm{ml}$. Este análisis se llevó a cabo en un cromatógrafo de gases Agilent Technologies 7890 que se acopló a un espectrómetro de masas Agilent Technologies 5975C, equipado con un puerto de inyección split/ splitlees (relación 1:20), un inyector automático Agilent 7863 y un sistema de datos HP Chem Station. Para la separación de las mezclas, se utilizó una columna capilar DB-5MS (30 m x 0,25 mm, D. I. x 0,25 $\mu \mathrm{m}$, df). La temperatura del horno $70{ }^{\circ} \mathrm{C}$ por $1 \mathrm{~min}$, desde $10{ }^{\circ} \mathrm{C} / \mathrm{min}$ hasta $120{ }^{\circ} \mathrm{C}$ por $15 \mathrm{~min}$, desde $5^{\circ} \mathrm{C} / \mathrm{min}$ hasta $150^{\circ} \mathrm{C}$ por $20 \mathrm{~min}$, y finalmente desde $25^{\circ} \mathrm{C} / \mathrm{min}$ hasta $300^{\circ} \mathrm{C}$ manteniéndolo por $1 \mathrm{~min}$. La temperatura de la 
cámara de ionización fue de $250^{\circ} \mathrm{C}$. El gas de arrastre empleado fue helio $(99,995 \%)$. Los espectros de masas y corrientes iónicas reconstruidas se obtuvieron por medio de barrido automático de frecuencia (full scan), a 4,75 scan s$^{-1}$, en el rango de masas $m / z$ 35-500. La temperatura de la línea de transferencia fue de $280^{\circ} \mathrm{C}$.

La identificación de los compuestos se realizó mediante la comparación de los tiempos de retención y los patrones de fragmentación representados en los espectros de masas experimentales con los encontrados en las bases de datos o librerías (database $\backslash$ NIST versión 2).

\subsection{Resultados y discusión}

\subsubsection{Características físicas del aceite esencial de orégano}

En la tabla 1 se muestran las condiciones, rendimientos y propiedades físicas del aceite esencial de orégano obtenido por destilación con arrastre de vapor de agua (en la tabla se presentan los valores promedio de las repeticiones).

Tabla 1

Características del aceite esencial de orégano

\begin{tabular}{ll}
\hline \multicolumn{1}{c}{ Muestra } & Origanum vulgare \\
\hline Tiempo de destilación & $60 \mathrm{~min}$ \\
Rendimiento & $2,02 \%$ \\
Densidad específica $a 20{ }^{\circ} \mathrm{C}$ & $0,9132 \mathrm{~g} / \mathrm{ml}$ \\
Índice de refracción & 1,475 \\
Solubilidad en alcohol & $2 / 75$ \\
Color $\left(\mathrm{CIE} \mathrm{L}^{*}, \mathrm{a}^{*}, \mathrm{~b}^{*}\right)$ & $\mathrm{L}^{*} 60,51 ; \mathrm{a}^{*}-2,83, \mathrm{~b}^{*} 14,31$ \\
\hline
\end{tabular}

Elaboración propia

Según lo obtenido, la densidad fue de $0,9132 \mathrm{~g} / \mathrm{ml}$ y el índice de refracción de 1,475; este último es ligeramente menor que el reportado por algunos autores (Poulose y Croteau, 1978). De acuerdo con investigaciones anteriores, un menor valor del índice de refracción y densidad está relacionado con una menor cantidad de fenoles (Hüsnü, Başer y Demirci, 2007). 


\subsubsection{Análisis cromatográfico (GC-MS) del aceite esencial de orégano}

Mediante el GC-MS se identificaron 33 compuestos químicos en las hojas de orégano (Origanum vulgare spp.). El cromatograma se presenta en la figura 2; y en la tabla 2 se mencionan los compuestos, tiempo de retención en minutos y el porcentaje con respecto a la muestra.

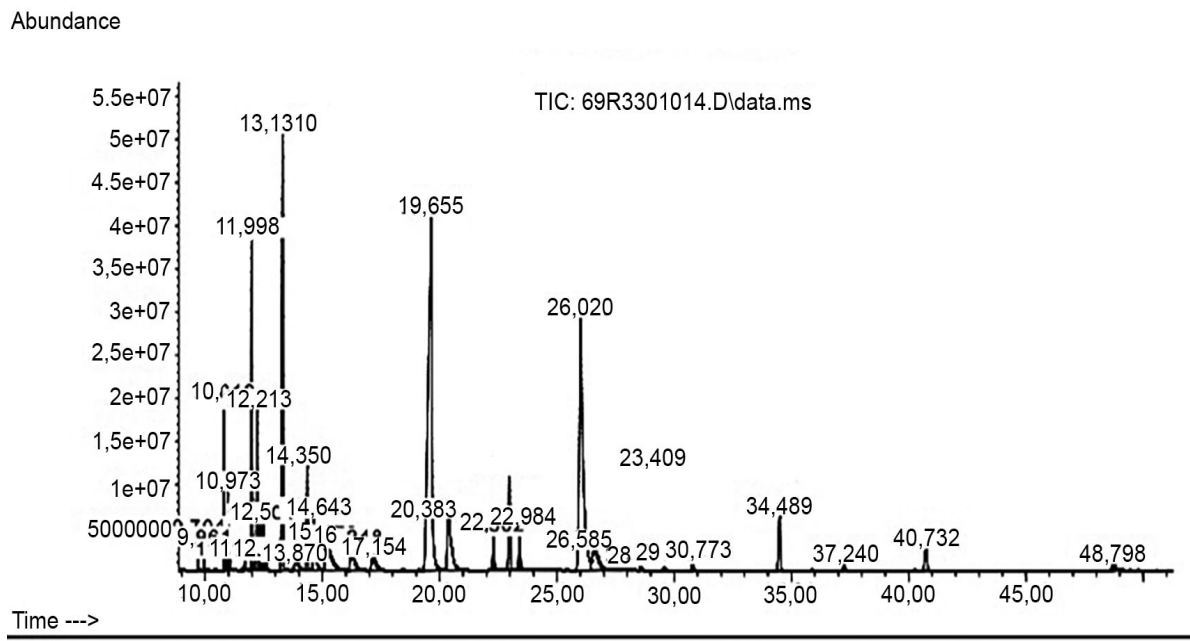

Figura 3. Cromatograma del aceite esencial de orégano Elaboración propia

Tabla 2

Composición química del aceite esencial de orégano (Origanum vulgare spp.)

\begin{tabular}{clcc}
\hline Número & $\begin{array}{r}\text { Nombre del } \\
\text { compuesto }\end{array}$ & $\mathbf{t}_{\mathrm{R}}(\mathrm{min})$ & $\begin{array}{c}\text { Porcentaje } \\
\text { en la muestra } \\
\text { (áreas relativas) }\end{array}$ \\
\hline 1 & a-tujeno & 9,70 & 0,51 \\
2 & a-pineno & 9,96 & 0,36 \\
3 & Sabineno & 10,82 & 2,81 \\
4 & 3-mirceno & 10,97 & 1,25 \\
5 & 3-pineno & 11,07 & 0,18 \\
6 & a-felandreno & 11,69 & 0,23 \\
7 & 2-careno & 12,00 & 6,53 \\
8 & $m$-Cimeno & 12,21 & 3,27 \\
\hline
\end{tabular}

(Continúa) 
(Continuación)

\begin{tabular}{|c|c|c|c|}
\hline Número & Nombre del compuesto & $t_{R}(\min )$ & $\begin{array}{c}\text { Porcentaje } \\
\text { en la muestra } \\
\text { (áreas relativas) }\end{array}$ \\
\hline 9 & D-limoneno & 12,37 & 0,85 \\
\hline 10 & $\beta$-felandreno & 12,50 & 0,91 \\
\hline 11 & Cis- $\beta$-ocimeno & 12,62 & 0,19 \\
\hline 12 & y-terpineno & 13,31 & 11,77 \\
\hline 13 & $\begin{array}{l}(1 \alpha, 2 \beta, 5 \alpha) \text { - 2-metil-5- } \\
\text { (1-metiletil) - biciclo[3.1.0] } \\
\text { hexan-2-ol. }\end{array}$ & 13,87 & 0,58 \\
\hline 14 & a-terpinoleno & 14,35 & 2,26 \\
\hline 15 & $\beta$-linalool & 14,64 & 2,25 \\
\hline 16 & cis- $\beta$-terpineol & 15,18 & 2,92 \\
\hline 17 & $\begin{array}{l}\text { Trans-1-metil-4-(1-metiletil) } \\
\text { 2-ciclohexen-1-ol }\end{array}$ & 16,25 & 1,54 \\
\hline 18 & $\begin{array}{l}\text { cis-1-metil-4-(1-metiletil) } \\
\text { 2-ciclohexen-1-ol }\end{array}$ & 17,15 & 1,05 \\
\hline 19 & L-4-terpineol & 19,65 & 26,56 \\
\hline 20 & a-terpineol & 20,38 & 4,08 \\
\hline 21 & Timol-metil éter & 22,30 & 0,99 \\
\hline 22 & $\begin{array}{l}\text { 1-metoxi-4-metil-2- } \\
\text { (1-metiletil)-benceno }\end{array}$ & 22,99 & 2,73 \\
\hline 23 & $\begin{array}{l}\text { 1,6-octadien-3-ol, } \\
\text { 3,7-dimethyl-, 2- } \\
\text { aminobenzoate }\end{array}$ & 23,41 & 1,17 \\
\hline 24 & Timol & 26,02 & 18,80 \\
\hline 25 & Carvacrol & 26,58 & 2,24 \\
\hline 26 & Elixeno & 28,57 & 0,10 \\
\hline 27 & Acetato de nerol & 29,58 & 0,16 \\
\hline 28 & Acetato de geraniol & 30,77 & 0,30 \\
\hline 29 & $\beta$-cariofileno & 34,49 & 1,87 \\
\hline 30 & a-cariofileno & 37,24 & 0,19 \\
\hline 31 & ס-elemeno & 40,73 & 0,95 \\
\hline 32 & (-)-spatulenol & 48,71 & 0,20 \\
\hline 33 & Óxido de cariofileno & 48,80 & 0,19 \\
\hline
\end{tabular}

Elaboración propia 
Los principales componentes del aceite fueron L-4-terpineol o terpinen-4-ol (26,56 \%), timol (18,80 \%) y carvacrol (2,24 \%), clasificados por orden de elución. Este resultado implica que el quimiotipo del AEO involucrado fue L-4-terpineol/timol. Al respecto, en muestras de orégano de provincias del sur de Argentina (Asensio, Grossob y Juliania, 2015) se extrajeron aceites esenciales con contenidos similares de L-4 terpineol y timol (11\% y $6 \%$, respectivamente), definidos como monoterpenos con cítricos, menta y notas de madera de coníferas. Este aceite es un potencial antioxidante y biocida, y son interesantes sus múltiples usos. Adicionalmente, se ha demostrado la actividad antidiabética de componentes como el timol y el carvacrol (Hyun, H. Kim y J. Kim, 2014; Sarikurkcua et al., 2015).

Govindarajan et al., (2016) realizaron un estudio en la India y hallaron que el carvacrol (38,3 \%) y el L-4-terpineol (28,70 \%) fueron los fenoles y monoterpenos mayoritarios; además, afirmaron que a partir de ellos podrían desarrollarse larvicidas naturales contra la malaria, la filariasis y la encefalitis de mosquitos. Por otro lado, es importante indicar que la presencia de timol en el aceite esencial de orégano ha sido investigada anteriormente (Banchio, Bogino, Zygadlo y Giordano, 2008; Muñoz et al., 2007) y por ello se conoce el efecto antimicrobiano y la actividad antirradicalaria que posee, e incluso su potencial aplicación en perfumería y cosmética (Acevedo et al., 2013).

Otros autores indican que generalmente en el aceite esencial de orégano existen componentes principales que determinan sus propiedades biológicas. Los componentes incluyen dos grupos de distinto origen biosintético (Bakkali, F. Averbeck, D. Averbeck y Idaomar, 2008): el grupo principal incluye terpenos y terpenoides; y el otro está formado por componentes aromáticos y alifáticos, todos caracterizados por su bajo peso molecular. Estos dos grupos se pueden clasificar en terpenoides e hidrocarburos no terpenoides (Hyun et al., 2014).

Las especies Origanum vulgare ssp. Vulgare y Virens (OVV) han sido mencionadas por ser ricas en compuestos sesquiterpenoides (germacrene D), y monoterpenos (terpinen-4-ol) (Sezik, Tumen, Kirimer, Ozek y Baser, 1993; Kula, Majda, Stoyanova y Georgiev, 2007; Dambolena et al., 2010); sin embargo, los quimiotipos frecuentemente determinados son carvacrol y timol. Los factores principales y secundarios, como la herencia genética, edad de las plantas y elementos ambientales, influyen en sus concentraciones dentro de una misma especia (Poulose y 
Croteau, 1978). Por esta razón, se da la diferencia en cuanto a sus componentes principales, incluso entre variedades de orégano de procedencias similares.

De acuerdo con la tabla 2, otros compuestos encontrados fueron $\gamma$-terpineno, 11,77\%; 2-careno, 6,53\%; m-cimeno, 3,27\%; sabineno, 2,81 $\%$; 1-metoxi-4-metil-2-(1-metil etil)-benceno, 2,7 3\%; $\infty$-terpinoleno, 2,26 $\%$; B-linalool, 2,25 \%; y carvacrol, 2,24 \%. La presencia de los compuestos minoritarios ya indicados coincide con la información transmitida por otros autores (Govindarajan et al., 2016), pero se han encontrado algunas diferencias cuantitativas en su composición. Estas pueden deberse al grado de sensibilidad analítica con que se trabaje, pues se ha encontrado alguna variación entre dos partidas de un mismo aceite esencial a causa de la época de cosecha, el año, el método de extracción y las condiciones de almacenamiento. De igual forma, variables como las condiciones geobotánicas, tipo de suelo, época de recolección y edad de la planta, entre otros (Crocoll et al., 2010), indican que las diferencias cuantitativas pueden atribuirse a los métodos de obtención, así como fecha y tiempo transcurrido entre la recolección y el proceso de obtención del aceite esencial (Farías et al., 2010).

\section{CONCLUSIONES}

En esta investigación, los monoterpenos y los fenoles fueron los grupos de terpenos más abundantes, estudiados por sus propiedades antioxidantes. Otros componentes encontrados fueron terpineno-4-ol, timol y carvacrol. Estos, debido a su poder antirradicalario (antioxidante) y biocida, ponen de manifiesto el potencial prometedor del aceite esencial de orégano en productos veterinarios, farmacéuticos y medicinales.

\section{REFERENCIAS}

Acevedo, D., Navarro, M., y Monroy, L. (2013). Composición química del aceite esencial de hojas de orégano (Origanum vulgare). Información Tecnológica, (24), 43-48.

Asensio, C., Grossob, N., y Juliania, H. (2015). Quality Characters, Chemical Composition and Biological Activities of Oregano (Origanum spp.). Essential Oils from Central and Southern Argentina. Industrial Crops and Products, (63), 203-213. 
Bakkali, F., Averbeck, F., Averbeck, D., y Idaomar, M. (2008). Biological Effects of Essential Oils - A Review. Food and Chemical Toxicology, (46), 446 -475.

Banchio, E., Bogino, P., Zygadlo, J., y Giordano, W. (2008). Plant Growth Promoting Rhizobacteria Improve Growth and Essential Oil Yield in Origanum majorana L. Biochem Syst Ecology, 36(10), 766-771.

Crocoll, Ch., Asbach, J., Novak, J., y Gershenzon, J. (2010). Terpene Synthases of Oregano (Origanum L.) and their Roles in the Pathway and Regulation of Biosynthesis. Plant Mol. Biol, 73(6), 587-603.

Dambolena, J., Zunino, M., Lucini, E., Olmedo, R., Banchio, E., Bima, P., y Zygadlo, J. (2010). Phenolic Content, Radical Scavenging Properties and Essential Oils Composition of Origanum Species from Different Populations. Journal of Agricultural and Food Chemistry, (58), 1115-1120.

Farías, G., Brutti, O., Grau, G., Di-Leo-Lira, R., Rettad, D., Van-Baren, C., ... y Bandoni, A. (2010). BandoniA. Morphological, Yielding and Quality Descriptors of Four Clones of Origanum spp. (Lamiaceae) from the Argentine Littoral Region Germplasm Bank. Industrial Crops and Products, (32), 472-480.

Govindarajan, M., Rajeswarya, M., Hotib, S. L., y Benellic G. (2016). Larvicidal Potential of Carvacrol and Terpinen-4-Ol from the Essential Oil of Origanum vulgare (Lamiaceae) Against Anopheles stephensi, Anopheles subpictus, Culex quinquefasciatus and Culex tritaeniorhynchus (Diptera: Culicidae). Research in Veterinary Science, (104), 77-82.

Hüsnü, K., Başer, C., y Demirci, F. (2007). Chemistry of Essential Oils. Flavours and Fragrances, (1), 43-86.

Hyun, T., Kim, H., y Kim, J. (2014). Antioxidant and Antidiabetic Activity of Thymus quinquecostatus Celak. Industrial Crops and Products, (52), 611-616.

Kula, J., Majda, T., Stoyanova, A., y Georgiev, E. (2007). Chemical Composition of Origanum vulgare spp. Essential Oil from Bulgaria. J. Essent. Oil-Bea. Plants, (10), 215-220.

Maximixe. (2007). Informe de mercado: orégano. Lima: Autor. 
Muñoz, A., Castañeda, M., Blanco, K., Cárdenas, C., Reyes, J., Kouznetsov, V., y Stashenko, E. (2007). Composición y capacidad antioxidante de especies aromáticas y medicinales con alto contenido de timol y carvacrol. Scientia et Technica, 13(33), 125128.

Poulose, A. J., y Croteau, R. (1978). Biosynthesis of Aromatic Monoterpenes. Conversion of Gamma-terpinene to P-cymene and Thymol in Thymus vulgaris L. Arch. Biochem. Biophys, 187(2), 307-314.

Sarikurkcua, C., Zengin, G., Oskayc, M., Uysalb, M., Ceylanb, R., y Aktumsekb, A. (2015). Composition, Antioxidant, Antimicrobial and Enzyme Inhibition Activities of Two Origanum vulgare Subspecies (Subsp. vulgare and Subsp. hirtum) Essential Oils. Industrial Crops and Products, (70), 178-184.

Sezik, E., Tumen, G., Kirimer, N., Ozek, T., y Baser, K. (1993). Essential Oil Composition of Four Origanum vulgare Subspecies of Anatolian Origin. J. Essent. Oil Res, (5), 425-431. 
